\title{
Natural history of HIV infection in older patients
}

\author{
G Orlando \\ From de Senectute: Age and Health Forum \\ Catanzaro, Italy. 5-7 December 2009
}

\section{Background}

The decreased mortality rate obtained with antiretrovirals (ARVs) induced a shift of HIV infection toward a chronic condition and increased the fraction of patients aged $>50$ years. Senior patients can be included in three subgroups with different clinical problems.

Long term exposure to ARVs give rise to the main clinical problems. Several studies recognize age as an independent risk factor for acute and chronic toxicities probably because of an additive mechanism with age related health problems (metabolic syndrome, cardiovascular diseases, endocrine-metabolic, liver, renal, bone and central or peripheral nervous system impairment. Among 1220 HIV we observed a higher rate of switching/discontinuation due to toxic effects in the elderly (Figure 1).Interaction between ARVs and several drugs widely used in the elderly $\left(\mathrm{H}_{2}\right.$-Antagonists, Pump-
Proton-Inhibitors, Benzodiazepines, Calcium-ChannelBlockers, Hormonal-Contraceptives, HMG-CoA-Reductase-Inhibitors, Phosphodiesterase-Type-5-Inhibitors, and Oral-Anticoagulants) are reported to and can influence both treatment strategy and effectiveness in older patients.

\section{Late primary infection}

Acute infections are hardly recognized but, since most HIV infections acquired in advanced ages are sexually transmitted, association with other STI is expected in this subset of patients who are not aware of own their risk, mode of HIV transmission and seem poorly reached by health education programs on safe sexual behaviour. Post-menopausal women are at a higher risk of infection due to hormonal mucosal changes and decreased use of condoms.

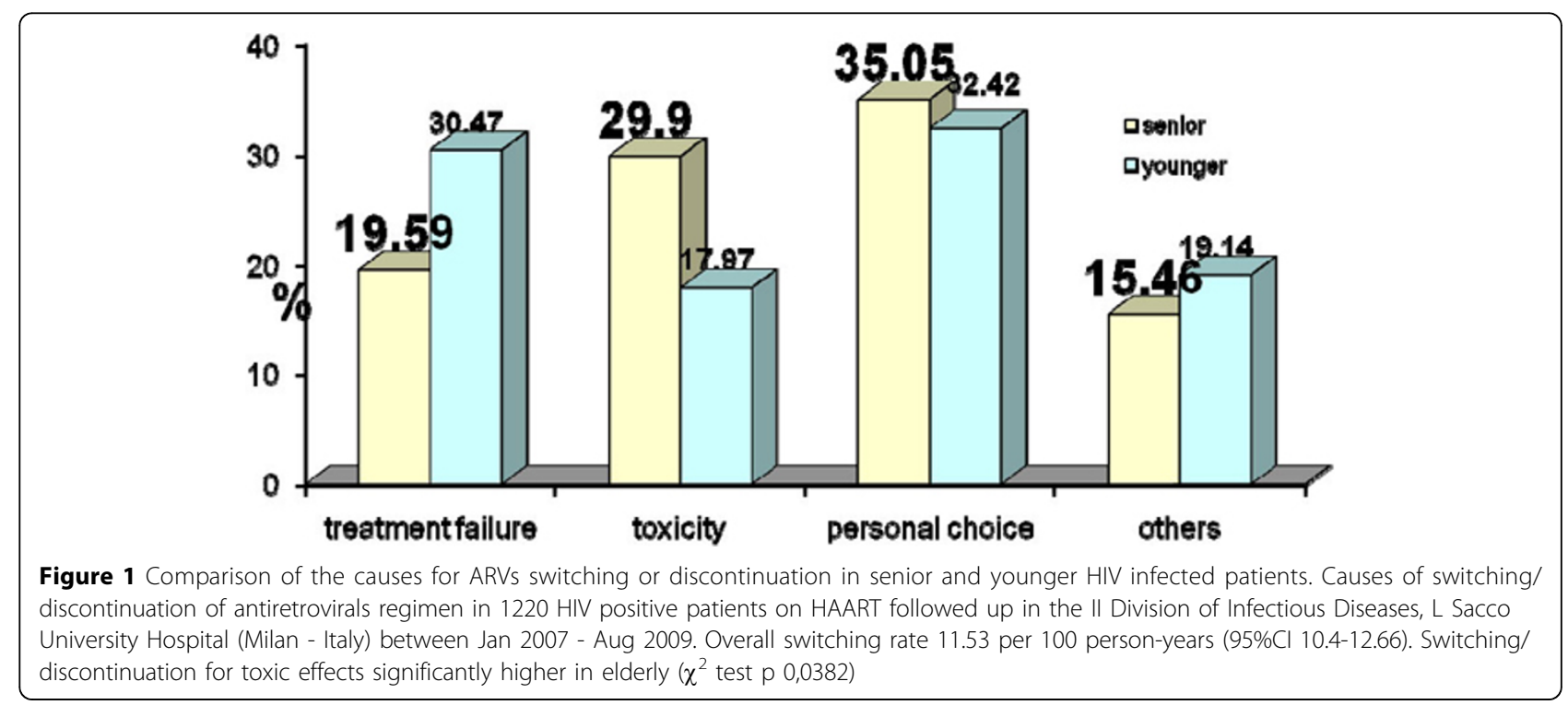

STD Unit, Infectious Diseases II, Infectious Diseases Department; L Sacco

University Hospital, Milan, Italy 


\section{Diagnostic delay}

Older persons are not perceived to be at risk from HIV by their physicians so that diagnosis is delayed. Moreover some AIDS related diseases (acute or chronic lung diseases, anaemia, cancers, peripheral/central neurologic disorders, herpes zoster, tuberculosis) could be attributed to advanced age. A large proportion of senior patients arrive at medical attention as AIDS presenters or late presenters, a factor which is associated with poorer prognosis and higher risk of disease transmission. AIDS defining diseases are very close to those found in the younger population, Pneumocystis jiroveci pneumonia being the most common occurring disease in AIDS presenters. The increased hospitalization rate among senior patients between 1996 and 2000 were mainly due to cerebrovascular and ischemic heart diseases. Among causes of death a higher proportion of non-AIDS related factors sepsis, non-AIDS malignancies and heart or vascular diseases have been described.

Clinicians should now be aware of the new challenges in management of HIV infected patients related to long term treatments, increased median age of the patients and appearance of age-related diseases.

Published: 19 May 2010

\section{Reference}

1. Orlando $G$, et al: Antiretroviral treatment and age-related comorbidities in a cohort of older HIV-infected pts. HIV Med. 2006, 7(8):549-57. 